\title{
Isolation and functional expression of a novel lipase gene isolated directly from oil-contaminated soil
}

\author{
Kaijing Zuo', Lida Zhang ${ }^{1}$, Hongyan Yao' and Jin Wang ${ }^{2 \bowtie}$ \\ IPlant Biotechnology Center, Fudan-SJTU-Nottingham Plant Biotechnology R\&D Center, College of Agriculture and Life Science, Shanghai Jiao- \\ tong University, Shanghai, China; 2Key Laboratory for Nuclear Waste Treatment \& Environmental Safety (SWUST), College of Science, Technology \\ and Industry for National Defense, Southwest University of Science and Technology, Mianyang, Sichuan, China
}

\begin{abstract}
A lipase gene SR1 encoding an extracellular lipase was isolated from oil-contaminated soil and expressed in Escherichia coli. The gene contained a 1845 -bp reading frame and encoded a 615 -amino-acid lipase protein. The mature part of the lipase was expressed with an N-terminal histidine tag in E. coli BL21, purified and characterized biochemically. The results showed that the purified lipase combines the properties of Pseudomonas chlororaphis and other Serratia lipases characterized so far. Its optimum $\mathrm{pH}$ and temperature for hydrolysis activity was $\mathrm{pH}$ 5.5-8.0 and $37^{\circ} \mathrm{C}$ respectively. The enzyme showed high preference for short chain substrates $(556.3 \pm 2.8$ $\mathrm{U} / \mu \mathrm{g}$ for (10 fatty acid oil) and surprisingly it also displayed high activity for long-chain fatty acid. The deduced lipase SR1 protein is probably from Serratia, and is organized as a prepro-protein and belongs to the GXSXG lipase family.
\end{abstract}

Keywords: lipase, gene cloning, hydrolysis activity, meta-genomic library

Received: 15 October, 2009; revised: 14 May, 2010; accepted: 19 August, 2010; available on-line: 02 October, 2010

\section{INTRODUCTION}

Lipases (EC 3.1.1.3) play an important role in lipid metabolism as biocatalysts for lipolytic reactions. Many lipases have been purified and cloned from bacteria and fungi such as Burkholderia glumae, Candida cylindracea, C. rugosa, C. albicans, C. deformans, Geotrichum candidum, Trichosporon fermentans, Pseudomonas fragi, and Staphylococcus aureus (Gilbert et al., 1991; Akastuka et al., 1994; Lee et al., 1994; Choo et al., 1998; Gupta et al., 2004; Kim et al., 2007; Shu et al., 2007; Liu et al., 2008). They can catalyze lipolytic, alcoholysis, acidolysis, esterification or trans-esterification reactions under different conditions (Yan et al., 2007). They exhibit broad substrate specificity and degrade acyl $p$-nitrophenyl esters, tweens, and phospholipids with positional, stereo and chain-length selectivity (Jaeger et al., 1994; 1999). Lipases have been widely used in many fields including food processes, biomedical or chemical industry, leather industry and cosmetics industry as well as environment management (Jaeger et al., 1994; 1998; 1999; Hanson et al., 2006).

Lipases from extreme environments have recently attracted attention because they are heat/cold-tolerant, show organic solvents tolerance and special catalytic activities (Feller et al., 1991; Jiang et al., 2006; Liu et al., 2008; Kiran et al., 2008). Mazzafera et al. (1996) have re- ported that a lipase from Serratia marcescens isolated from soil under coffee cultivation can degrade caffeine and related methylxanthines. Several lipases (such as those from Yarrowia lipolytica CL180 or Aureobasidium pullulans HN2-3) from cold marine environment displayed high lipolytic activity (Kim et al., 2007; Liu et al., 2008). In salmon grease-contaminated soil, most lipases originated from the Myroide, Arthrobacter, Bacillus and Serratia genus (Ciudad et al., 2008). Margesin et al. (1999) have found that monitoring of soil microbial lipase activity is a valuable indicator of diesel oil biodegradation in freshly contaminated soils. These results indicate that there is a great diversity of lipases in oil-contaminated environment and these lipases could be used in oil biodegradation (Jaeger et al., 1999; Cervantes-González et al., 2004).

In this report, we described the cloning of a lipase gene from oil-contaminated soil and the purification of recombinant his-tagged mature form of the lipase. Biochemical characterization of this lipase revealed that its properties differ markedly from those of other reported lipases. It had a high hydrolytic activity towards peanut oil without trans-esterification activity. Homology alignment showed high homology of this lipase with other Serratia lipases except ten nucleotide substitutions. This lipase gene probably originated from Serratia and the encoded enzyme could find use in environment management.

\section{MATERIALS AND METHODS}

Bacterial strains. Escherichia coli $\mathrm{DH} 5 \alpha$ and $\mathrm{DH} 10 \mathrm{~B}$ were used for cloning of the lipase gene. The plasmids pMD18-T and pET-32a were used as gene cloning and protein expression vectors, respectively. For the construction of expression plasmids, E. coli strain BL21 was used.

Lipase gene cloning and sequence analysis. Oilcontaminated soil was collected from the ground of a waste oil and sewage treatment area in the cafeteria of Shanghai Jiaotong University (Shanghai, China). Meta-genomic DNA of oil-contaminated soil was isolated according to the method of Gabor (2004). Crude DNA was purified by agarose gel electrophoresis using QIAEXII Gel Extraction Kit (USA). Purified DNA was then partially digested with Sau3AI (Promega, Mannheim, Germany) and separated by electrophoresis on

\footnotetext{
e-mail: wjdsz@vip.sina.com.cn
} 
$1 \%$ agarose gel. DNA fragments of $2-5 \mathrm{~kb}$ were then ligated into BamHI digested pMD18 (Takara, Japan) plasmid DNA using T4 DNA Ligase (Promega, USA). The recombinant plasmids were electroporated into $E$. coli $\mathrm{DH} 10 \mathrm{~B}$ to generate a meta-genomic library (Henne et al., 2000; Helen et al., 2005; Lee et al., 2006).

The Gene Images random priming labeling kit and Gene Images CDP-Star detection kit (Amersham Pharmarcia, USA) were used for probe labeling and library screening. A fragment derived from conserved regions (GXSXG, GDSL, 200 bp) of a multiple alignment of different bacterial lipases was used as the probe to screen the DNA library. The insert of the plasmid isolated was sequenced with M13 forward and reverse primers. The entire nucleotide sequence of the lipase gene, named as SR1 has been deposited in the GenBank database and assigned the Accession No. bankit 1162518. The putative protein and DNA sequence of the SR1 gene was blasted on the web (http://ncbi.nlm.nih.gov). The alignment analysis was performed with ClustalW (Thompson et al., 1994).

Protein expression and purification. To characterize the putative lipase encoded by the SR1 gene, it was expressed in E. coli BL21. Candida antartica lipase B (CALB) has been employed in organic synthesis with a broad range of applications (Anderson et al., 1998). So, the $C A L B$ gene was used as a control. The open reading frame of the lipase gene was amplified from $p M D 18-T$. The PCR product was digested with $\mathrm{NcoI} / \mathrm{EcoRI}$ and subcloned into the plasmid pET32-a. The plasmid of a positive clone was then transformed into E. coli BL21.

Lipase activity was detected by using rhodamine Btriolein agar plates by directly applying $4 \mathrm{mM}$ IPTGinduced cells to the plates with $50 \mathrm{mg} / 1$ ampicillin to screen for lipase protein expressing clones. Lipase activity was observed when the colonies became deeply red after $24 \mathrm{~h}$ of cultivation at $37^{\circ} \mathrm{C}$.

A positive clone was inoculated into liquid LB with $50 \mathrm{mg} / 1$ ampicillin. Isopropyl- $\beta$-D-thiogalactopyranoside (final concentration $4 \mathrm{mM}$ ) was added when $\mathrm{OD}_{600}$ of cultures reached 0.6. The cells were further cultured for $3 \mathrm{~h}$ and harvested by centrifugation at $4000 \mathrm{rpm}$ at $4^{\circ} \mathrm{C}$ for $10 \mathrm{~min}$. The pellets were re-suspended in 50 $\mathrm{mM}$ Tris/ $\mathrm{HCl}(\mathrm{pH} 7.5)$ containing $0.5 \mathrm{M} \mathrm{NaCl}$ and 1 $\mathrm{mM}$ phenylmethylsulfonylfluoride and lysed by sonication. Following centrifugation at $15000 \mathrm{rpm}$ at $4^{\circ} \mathrm{C}$ for $30 \mathrm{~min}$, the supernatant was analyzed by sodium dodecyl sulfate-polyacrylamide gel electrophoresis to assess the expression of lipase protein. The supernatant of a highly expressing clone was then loaded onto a His-tag Sepharose-Fast-Flow gel column (Amersham-Pharmacia Biotech, USA) and washed with a gradient of $100 \mathrm{mM}$ to $1 \mathrm{M}$ wash solution to elute pure recombinant protein for activity analysis.

Analysis of enzyme activities. Trans-esterification assay of lipase protein: The trans-esterification activity towards soybean oil was assayed in a reaction containing $0.339 \mathrm{~g}$ of soybean oil and $0.034 \mathrm{~g}$ of enzyme; $3.6 \mathrm{ml}$ n-heptane and $48.5 \mu \mathrm{l}$ methanol was added to one-three volume by batch every $6 \mathrm{~h}$. The reaction was performed at $37^{\circ} \mathrm{C}$ for $24 \mathrm{~h}$ with 150 -rpm shaking (Freedman et al., 1984).

To follow the trans-esterification reaction, the fatty acid methyl ester (FAME) products were analyzed by an Agilent 6890N gas chromatograph equipped with an HP-5 5\% phenyl methyl siloxane capillary column (30 $\mathrm{m} \times 0.32 \mathrm{~mm} \times 0.25 \mu \mathrm{m})$, an automatic injector 7376 and a FID detector (Palo Alto, CA, USA). The injector and detector temperatures were $180^{\circ} \mathrm{C}$ and $300^{\circ} \mathrm{C}$, respectively. The sample analyses were performed at least three times.

Lipase hydrolysis activity analysis. Lipase hydrolysis activity was measured according to the method described by Winkler and Stuckmann (1979). p-Nitrophenyl caprate was the substrate in this study. The assay mixture contained $89 \mu \mathrm{l}$ of Tris/ $\mathrm{HCl}$ buffer $(50 \mathrm{mM}, \mathrm{pH} 7.5$ ), $10 \mu$ l of substrate solution $(25 \mathrm{mM}$ p-nitrophenyl caprate in DMSO), and $1 \mu$ l of enzyme solution $(0.001 \mathrm{mg})$. The reaction time was allowed to proceed for $10 \mathrm{~min}$ and was then stopped by adding $100 \mu \mathrm{l}$ of ethanol. The $A_{410}$ of liberated $p$-nitrophenol was measured with $p$-nitrophenol as a standard. One unit was defined as the mount of enzyme required to release $1 \mu \mathrm{mol}$ of $p$-nitrophenol per min under assay conditions (Luisa-Rúa et al., 1997).

The substrate specificity of the lipase towards different $p$-nitrophenyl esters ( $p$ NP-) was determined by a spectrophotometric method with $p$-nitrophenyl caprate, laurate, myristate, palmitate and stearate as substrates. Liberation of $p \mathrm{NP}$ was measured at room temperature by reading the absorbance at $410 \mathrm{~nm}$ (Schmidt-Dannert et al., 1994; Zhang et al., 2003).

pH and thermo-stability analysis of SR1 enzyme. The effects of $\mathrm{pH}$ on the recombinant lipase activity were determined by incubating the recombinant enzyme between $\mathrm{pH}$ 4.0-10.0. The buffers used in this study were $0.1 \mathrm{M}$ citric acid/sodium citrate buffer $(\mathrm{pH} 4.0-$ 5.5), $0.1 \mathrm{M}$ potassium phosphate buffer ( $\mathrm{pH}$ 6.0-8.0), and $0.1 \mathrm{M}$ glycine/ $\mathrm{NaOH}$ buffer $(\mathrm{pH} 8.5-10)$. The recombinant enzyme had been incubated in the buffer for $2 \mathrm{~h}$ at $37^{\circ} \mathrm{C}$. After incubation, the reaction buffer was cooled on ice to determine the remaining enzyme activities. The remaining activities of the recombinant lipase were measured immediately after this treatment with the standard method as described above.

The optimal temperature for activity of the enzyme was determined at $25,30,35,40,50,60$ and $70^{\circ} \mathrm{C}$ in the same buffer at $\mathrm{pH}$ 7.5. The thermo-stability of the recombinant enzyme was tested by pre-incubating the enzyme at different temperatures for $30 \mathrm{~min}$. The residual enzyme activity was measured as described above.

\section{RESULTS}

\section{Lipase gene cloning from oil-containment soil}

In order to clone the lipase gene, oil-containment soil DNA was partially digested with Sau3AI which resulted in fragments ranging in size from 2 to $5 \mathrm{~kb}$ as determined by electrophoresis in $0.4 \%(\mathrm{w} / \mathrm{v})$ agarose gels. The digested DNA was then ligated with BamHI-digested pMD18 vector (Takara, Japan) to generate an environmental meta-genomic DNA library with $6.07 \times 10^{5}$ clones. Using lipase-homologous fragment as the probe to screen this library, a clone with a 2133 -bp insert containing a complete lipase gene was isolated after three rounds' of screening. By DNA sequencing, a 2133-nucleotide insert was determined (Fig. 1) and named SR1 gene.

\section{Sequence analysis of SR1 gene}

Bioinformatic analysis showed that the SR1 gene contained an open reading frame from nucleotide 132 to 1977, encoding a protein of 615 amino-acids (Fig. 1). A database comparison using this amino-acid sequence revealed a significant similarity with various Psendomonas 
132 atgrgtgattttt tat tatcagggcctgatgaagcgaaatccaaa

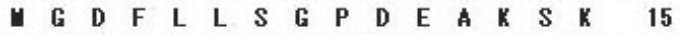

178 acgt tatt accgacgccatggczatt tccacet acgcct atcac $\begin{array}{llllllllllllllll}\text { T } & \mathbf{L} & \mathbf{F} & \mathbf{T} & \mathbf{D} & \mathbf{A} & \mathbf{U} & \mathbf{A} & \mathbf{I} & \mathrm{S} & \mathbf{T} & \mathbf{Y} & \mathbf{A} & \mathbf{Y} & \mathbf{H} & \mathbf{3 0}\end{array}$

223 aat at gat aatggttttgacgagggct atcacagctccggtttt M I I D N 268 ggcetgggct tgccet tcacgctggtgaccgcgctgataggcagc

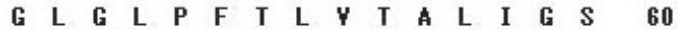
313 acgcaatct cagggt ggcetacceggt attccetggaat ccegac $\begin{array}{llllllllllllllll}\text { T } & \text { O } & \text { S } & \text { O } & \text { G } & \text { G } & \text { L } & \text { P } & \text { G } & \text { I } & \text { P } & \text { P } & \text { N } & \text { P } & \text { D } & 75\end{array}$ 358 tcggaacaagcggcgctggcagcggtt aat aacgccggct ggtca S E $\mathbf{Q}$ A A $403 \mathrm{ttgataagcgcagaccaactaggctatcagggcaaacggatgcc}$

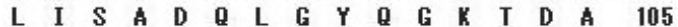
448 cgcggcacet at tacggt gaaacgt tcgget acaccacggcacag $\begin{array}{llllllllllllllll}R & G & T & Y & Y & G & E & T & F & G & Y & T & T & A & Q & 120\end{array}$ 493 get gaagtgctgggaaaat acgacagcgagggt aatctcaccggc $\begin{array}{llllllllllllllll}\text { A } & E & Y & L & G & K & Y & D & S & E & G & \text { N } & \text { L } & \text { T } & G & 135\end{array}$ 538 atcggcattgcttttcgeggcaccagcggcccgcgcgaatctctg

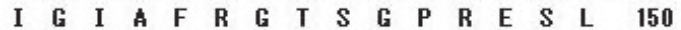
583 atcaccgat accatcggcgat ttggtcaat gacctgctggccggt $\begin{array}{llllllllllllllll}\text { I } & \text { T } & \text { D } & \text { T } & \text { I } & \text { G } & \text { D } & \text { L } & \text { Y } & \text { A } & \text { D } & \text { L } & \text { L } & \text { A } & \text { G } & 165\end{array}$ 628 ttcggcccgaat ggct at gccgat aact acagtctgaaagcettc

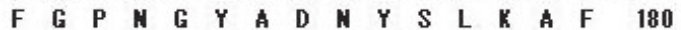
673 ggcaccctgctggaggat gtggccaaat tcgcccaggcgcacgg

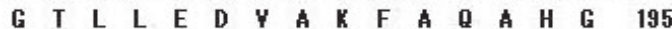
718 ctgagcggggat gacat accatcagt ggccat agcetcggcggc L $S$ S $\quad$ G 763 ctggccgtgaacagtatggczgczctcagcgacggcaat tggggg L A Y N S I A A L S D G N T G 225 808 ggct tct at gcgcagtccaact atgtcgcet ttget tetcct acc

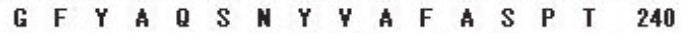
853 caat acgaaaccggcggcaaagtgatt aacgtcggt tacgaaaat $\begin{array}{llllllllllllllll}\text { Q } & \text { Y } & \text { E } & \text { T } & \text { G } & \text { G } & K & \text { Y } & \text { I } & \text { N } & \text { Y } & \text { G } & \text { Y } & \text { E } & \text { N } & 255\end{array}$ 898 gacccggtat tccgcgcgt tggacggt accaacgcgcacctctgc $\begin{array}{llllllllllllllll}\text { D } & P & \text { Y } & \text { F } & \text { R } & \text { A } & \text { L } & \text { D } & \text { G } & \text { T } & \text { N } & \text { A } & \text { H } & \text { L } & \text { C } & 270\end{array}$

943 aacgct gggcgtcat gacgcaccgcaagactccgccaccaacaac

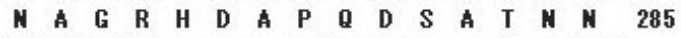

988 atcgtt aat tcaacgaccact at gcctccgccgcet ggaat att

I Y N
1033 ctgccet tctcgatcctcaat attccaacct ggt tatctcacctg $\begin{array}{llllllllllllllll}\text { L } & \text { P } & \text { F } & \text { S } & \text { I } & \text { L } & \text { H } & \text { I } & \text { P } & \text { I } & \text { I } & \text { L } & \text { S } & \text { H } & \text { L } & \mathbf{3 1 5}\end{array}$ 1078 ccgttcctt tatcaggacgggt tgatgcgggtat tgaat cagag $\begin{array}{llllllllllllllll}P & F & L & Y & \mathbf{Q} & \mathbf{D} & \mathbf{G} & \mathrm{L} & \text { H } & R & \text { Y } & \mathrm{L} & \text { N } & S & E & \mathbf{3 3 0}\end{array}$

1123 ttctactcgctgaccagcaaagactcgacggtgatcgtttccaac $\begin{array}{llllllllllllllll}F & Y & S & L & T & S & K & D & S & T & Y & I & Y & S & \text { N } & \mathbf{3 4 5}\end{array}$

1168 ctttctgacgtcacccgcggcaat acctgggtggaagacctcaac

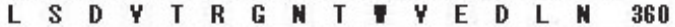

1213 cgcaacgccgagcaacacagcggcccaacct ttattgtcggcagc $\begin{array}{llllllllllllllll}\text { R } & \text { H } & \text { A } & \text { E } & \text { Q } & \text { H } & S & \text { G } & \text { P } & \text { T } & \text { F } & \text { I } & \text { Y } & \text { G } & S & 375\end{array}$

1258 gacggcaatgaccttatcaagggcggcactggaaat gat tatctc \begin{tabular}{lllllll|lllllllll} 
D & $G$ & N & $D$ & L & $I$ & $K$ & $G$ & G & T & G & A & D & $Y$ & L & 390
\end{tabular}

1303 gagggccgtgccggcaat gacacat tccgtgacgatggcggtt tt

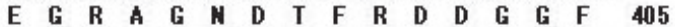

1348 aat at att tccggcggt gaaggccat aacaccctggat ttgcag \begin{tabular}{llll|llllllllllll} 
H & I & I & $S$ & $G$ & $G$ & G & $G$ & $H$ & N & T & L & $D$ & L & Q & 420
\end{tabular}

1393 cat gcat tgaaaacaccgaggtcgcgt acgacggcaat acgctc $\begin{array}{llllllllllllllll}\text { H } & \text { A } & \text { L } & K & \text { N } & \text { T } & \text { E } & \text { Y } & \text { A } & Y & \text { D } & \text { G } & \text { N } & \text { T } & \text { L } & 435\end{array}$

1438 tatt tgcgcgat gct gacggt ggcatcaccetggct aactcgatc $\begin{array}{llllllllllllllll}Y & L & R & D & \text { A } & \text { D } & \text { G } & \text { G } & \text { I } & \text { T } & \text { L } & \text { A } & \text { N } & S & \text { I } & \mathbf{4 5 0}\end{array}$

1483 ggcacgctgaaaagcaaagagtcgtcactgt tgattt tcaccaaa $\begin{array}{llllllllllllllll}\text { G } & T & L & K & S & K & E & S & S & L & L & I & F & T & K & 465\end{array}$

1528 gaggttgatcaccaggtgaccgacaacggct tgctttccaccaag $\begin{array}{llllllllllllllll}E & Y & D & H & \text { Q } & \text { Y } & \text { T } & \text { D } & \text { N } & \text { G } & \text { L } & \text { L } & S & \text { T } & K & 480\end{array}$

1573 gggctgaccgcet at gccagt tcggcaaacggcaccgczgccgat

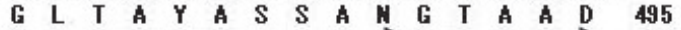

1618 gacgttctgactgct aaagagtccggct cetggctct tcggt tg $\begin{array}{llllllllllllllll}D & Y & L & T & A & K & E & S & G & S & & L & F & G & L & 510\end{array}$ 1663 gagggaaatgaccagctgtttggcggcaaaggcaat gacgtgttc $\begin{array}{lllllllllllllllll}\text { E } & \text { G } & \text { H } & D & \text { Q } & \text { L } & \text { F } & G & \text { G } & \text { K } & \text { G } & \text { A } & \text { D } & \text { Y } & \text { F } & 525\end{array}$

1708 gtcggcggcgcgggt aacgacgttatgcacagccagggtggcagc $\begin{array}{llllllllllllllll}Y & \text { G } & \text { G } & \text { A } & \text { G } & \text { N } & \text { D } & Y & \text { U } & \text { H } & S & \text { Q } & \text { G } & \text { G } & S & 540\end{array}$

1753 aatacet tcctgttcagtggcgact tcggtcaggatctgatt ac $\begin{array}{llllllllllllllll}\text { N } & \text { T } & F & L & F & S & G & D & F & G & \text { Q } & \text { D } & \text { L } & \text { I } & Y & 555\end{array}$

1798 ggt tatcaggcgcaggat aagctggtgtt atcggt accgaaggc $\begin{array}{llllllllllllllll}\text { G } & Y & \text { O } & \text { A } & \text { O } & \text { D } & K & \text { L } & \text { Y } & \text { F } & \text { I } & \text { G } & \text { T } & \text { E } & \text { G } & 570\end{array}$

1843 agcagctcgggcggt aat tatcgcgatttgtt tccgaggtgaac

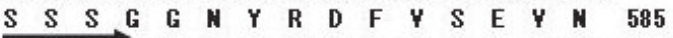

1888 gacaacct $g$ gtgtt aact ttggcggcagt acggtcact tagtc $\begin{array}{llllllllllllllll}\text { D } & \text { H } & L & \text { Y } & \text { F } & \text { N } & \text { F } & \text { G } & \text { G } & S & \text { T } & \text { Y } & \text { T } & \text { L } & \mathbf{Y} & \mathbf{6 0 0}\end{array}$

1933 gggatcggat tcgat agcetgtcggatggccaggtggtgctggcg tas

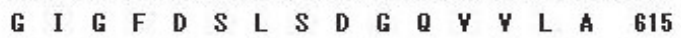

Figure 1. Full-length DNA sequence and deduced amino-acid sequences of SR1 lipase protein Nucleotide positions are given on the left side of the sequence in the $5^{\prime}$ to $3^{\prime}$ orientation. The start codon ATG and the stop codon TAG are in italics. The deduced amino-acid sequence is shown beneath the nucleotide sequence and the amino acids are numbered on the right side. The motif $G \times S \times G$ is underlined .... Another GSSSG similar to the motif $G \times S \times G$ is underlined with a thick arrow. Glycine-rich consensus motifs are boxed. A putative charged amphipathic region is double-arrowed. The sequence of SR1 lipase gene has been deposited at GenBank (Acc. No. bankit1162518).

and Serratia lipases. The highest overall identities were found with the lipase ZP_01534977 (Serratia proteamaculans), NP_929565 (Photorhabdus luminescens sub sp.), and AAD22743 (Psendomonas chlororaphis), sharing 98\%, 95\% and $90 \%$ identity, respectively. Compared with our previously reported SLlipA gene from Serratia liquefaciens S33 DB-1, the SR1 gene has six mutations at its N-terminal and four nucleotides mutations at its $\mathrm{C}$-terminal (Yao et al., 2008).

Direct alignment with other lipases revealed that SR1 lipase has no signal peptide at its N-terminus. The enzyme has four glycine-rich repeated motifs. This motif is always present in the RTX toxin family and in the proteins secreted by the class I secretion pathway ( $\mathrm{Li}$ et al., 1995). The amino-acid sequence derived from the nucleotide sequence contained the lipase-specific active-site consensus, Gly-X-Ser-X-Gly, found in the majority of bacterial and eukaryotic lipases. The Ser residue of this motif is the key element of the active site of fatty acidesterification lipases. Based on these characteristics, the
SR1 lipase was predicted to belong to the GXSXG family of lipases (Fig. 2).

\section{Expression and purification of SR1 lipase}

The open reading frame encoding mature SR1 lipase was amplified and cloned into pET-32a. The expression plasmid $p E T-32 a$ containing the SR1 gene was then transformed into $E$. coli BL21 strain to express the SR1 protein. The positive clones were confirmed by PCR. One hundred and twenty-eight positive clones were then in oculated into a selective medium to characterize SR1 lipase expression. The clones expressing active SR1 lipase were determined by a red halo on Rhodamine-Triglyceride-Agarose plate by adding $1 \mu$ l of supertant.

One clone which became deeply red after incubating for $24 \mathrm{~h}$ at $37^{\circ} \mathrm{C}$ was used in the subsequent analysis of enzyme activities (Fig. 3). After inducing with 4 mM IPTG for $4 \mathrm{~h}$, supernatants from different clones and a control (a clone with pET-32a) were used in protein analysis. Total proteins extracted from induced LB 
SR 1

A.AD 22743

A.AU25 837

ZP 0153497

A.A. 81002

NP 929565

P26504

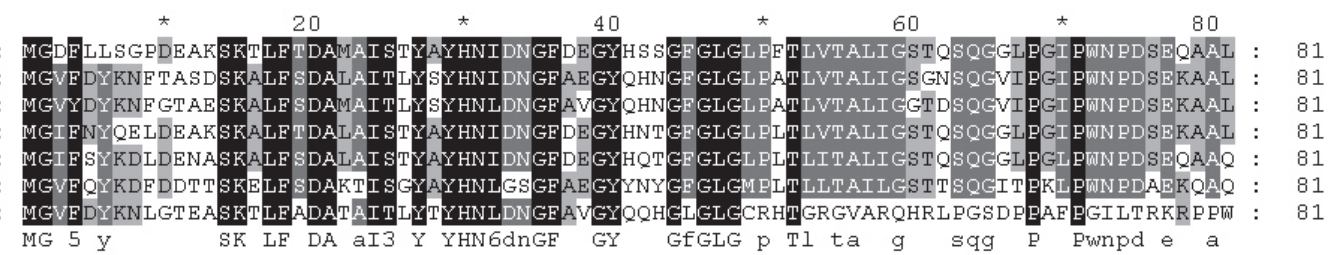

SR1

A.A.D 22743

A.AU25837

ZP 0153497

A.A. 81002

NP 929565

P2 $\overline{6} 504$

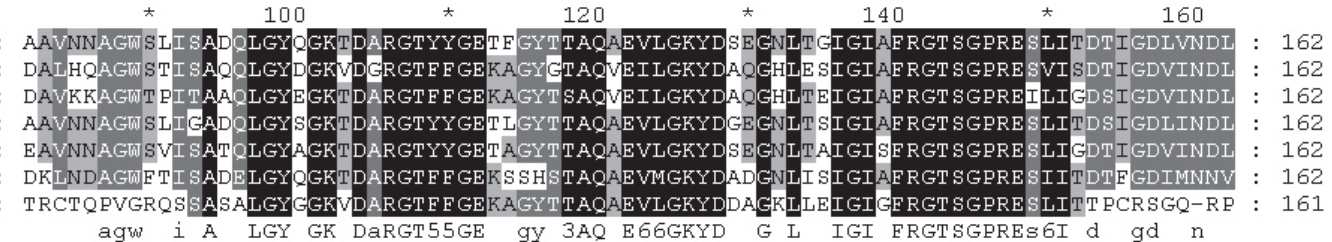

SR1

A.A.D22743

A.AU25837

ZP 0153497

A.A.A.81002

NP 929565

P26504

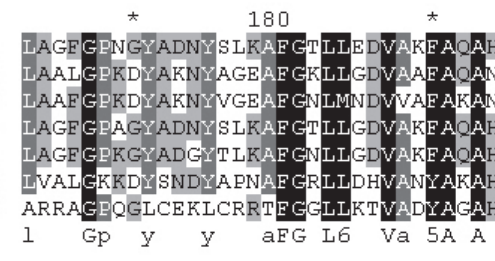

200

220

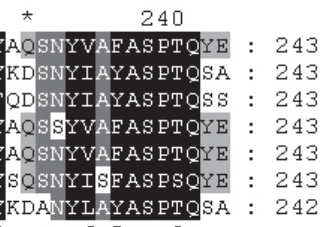

SR 1

A.AD 22743

A.AU25 837

ZP 0153497

A.A.81002

NP_929565

P2 $\overline{6} 504$
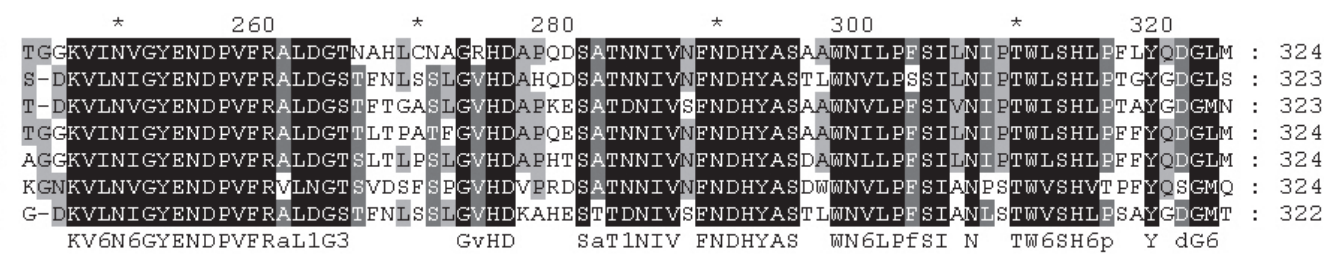

SR 1

A.A.D 22743

A.AU2 5837

ZP 0153497

A.A. 81002

NP 929565

P26504

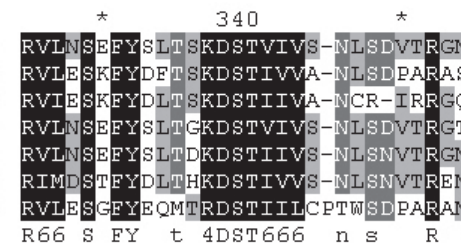

360
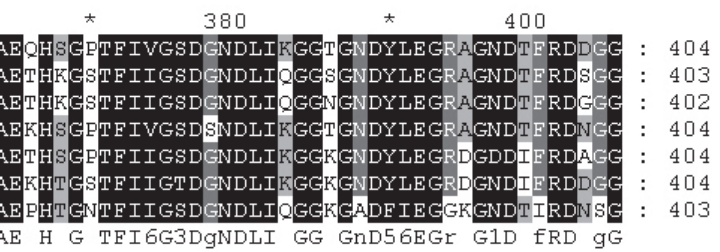

SR 1

A.AD 22743

A.AU25 837

ZP 0153497

A.A.A.81002

NP_929565

P2 $\overline{6} 504$

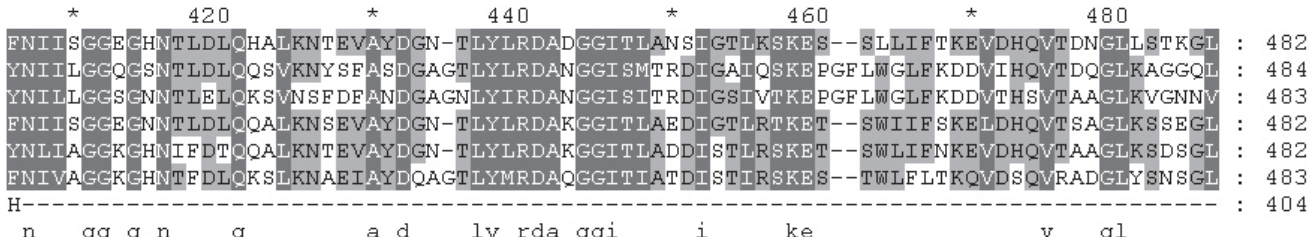

SR 1

A.AD 22743

A.A.U2 5837

ZP_0153497

A.A.A. 81002

NP_929565

P2 $\overline{6} 504$
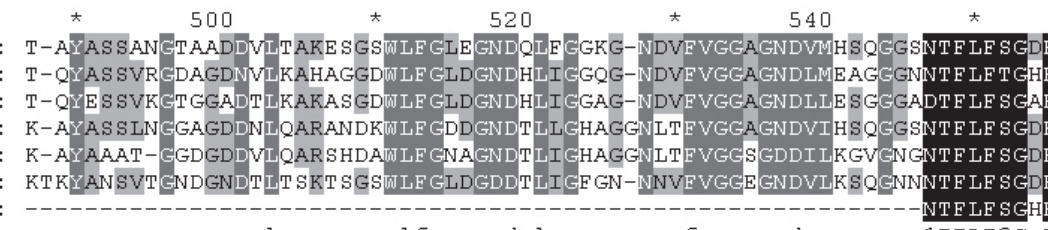

560

SR 1

A.A.D 22743

A.AU25 837

ZP_0153497

A.A.A.81002

NP_929565

P2 $\overline{6} 504$

wlfg $g \mathrm{~d} l \mathrm{~g}$

$n$ fvgg $g$ d

F LGDEGODIIYGYNKTD : 563

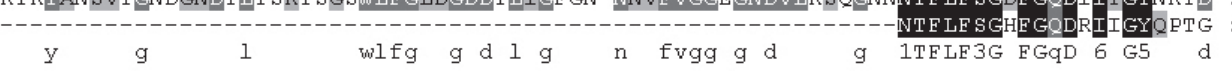

425

Figure 2. Alignment of amino-acid sequences of lipases from different bacterium and fungal accessions

The predicted amino-acid sequence of SR1 lipase was aligned with lipase polypeptide sequences from Serratia proteamaculans (ZP_01534977), S. marcescens (AAA81002), Photorhabdus luminescens sub sp. (NP_929565), Pseudomonas chlororaphis (AAD22743), P. fluorescens (AAU258370), Psychrobacter sp. PRwf-1 (ZP 01271540) using the Clustal multiple alignment program. Gaps to optimize alignments are designated by dashes (-). Asterisks $\left(^{*}\right)$ indicate consensus amino-acid identity among all organisms. Dots (. or :) indicate positions of conservative amino acid replacement. Black blocks show that these amino-acids were extremely conserved in all the proteins. Gray blocks show conserved amino acids. The numbers at the top or at the right column refer to the position in the amino-acid sequence. 


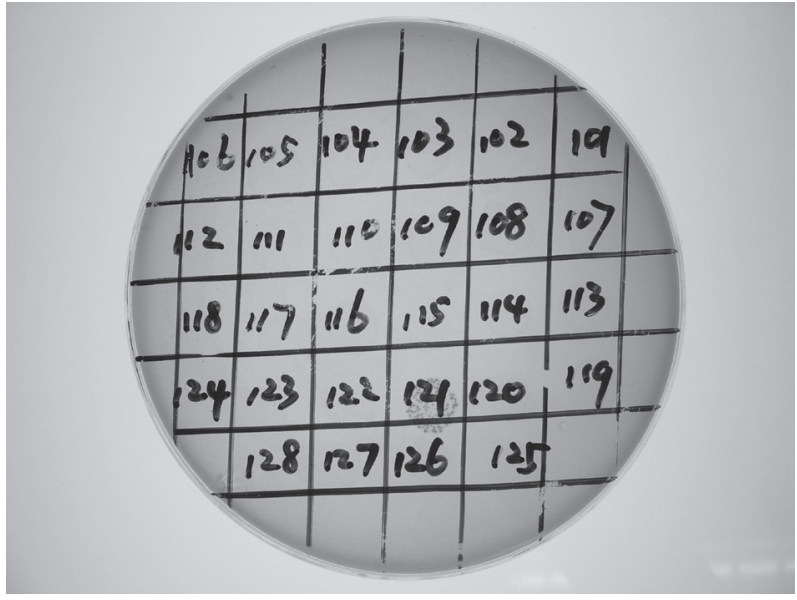

Figure 3. Rhodamine B-triglyceride agar plate showing different recombinant clones

Five microliter of culture supernatant (sterile filtered) induced by IPTG was poured into each preformed hole, and the plate was incubated at $37^{\circ} \mathrm{C}$ overnight. Deeply red halo (No.121) indicates the production of recombinant lipase.
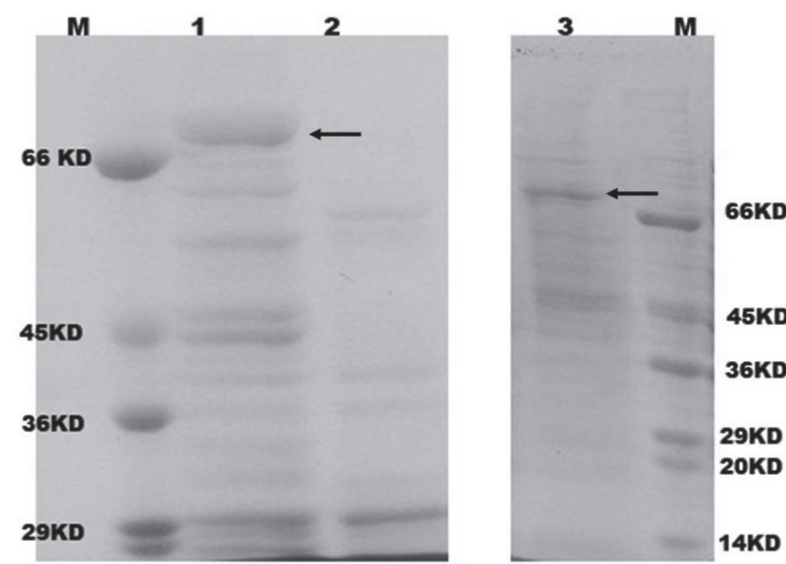

Figure 4. SDS/PAGE analysis of total secreted protein after induction with 4 mM IPTG

Culture supernatants were harvested at various time intervals, precipitated with $10 \%$ trichloroacetic acid, washed with acetone, freeze-dried and then analyzed by SDS/PAGE. The band intensities of Coomassie Blue-stained gel is shown. Lane $\mathrm{M}$, middle molecular mass protein markers (Watson, Shanghai, China). Lanes 1, 3, $4 \mathrm{~h}$ after induction with 4 mM IPTG. Lane 2, before induction.

were separated on a $15 \%$ SDS/PAGE gel. The results showed that those plasmids (containing SR1 gene) encoded the His-tagged mature lipase with a calculated molecular mass of $65 \mathrm{kDa}$ (Fig. 4).

Table 1. Spectrophotometric assay of substrate activity and specificity toward different $p$-nitrophenyl esters ( $p$ NP-).

\begin{tabular}{|c|c|c|c|c|c|}
\hline \multirow{2}{*}{$\begin{array}{l}\text { Enzyme } \\
(\mathrm{U} / \mu \mathrm{g})\end{array}$} & \multicolumn{5}{|c|}{ Substrate $^{a}$} \\
\hline & $p-\mathrm{NPC}_{10}$ & $p-\mathrm{NPC}_{12}$ & $p-\mathrm{NPC}_{14}$ & $p-\mathrm{NPC}_{16}$ & $p-\mathrm{NPC}_{18}$ \\
\hline CALB & $20.2 \pm 1.0$ & $19.3 \pm 0.8$ & $20.2 \pm 1.2$ & $19.6 \pm 0.7$ & $18.1 \pm 1.1$ \\
\hline SR1 & $556.3 \pm 2.8$ & $359.6 \pm 1.6$ & $325.4 \pm 3.0$ & $88.9 \pm 1.5$ & $26.0 \pm 1.1$ \\
\hline S33DB $1^{b}$ & $14.4 \pm 0.5$ & $19.8 \pm 0.6$ & $27.6 \pm 0.3$ & $17.3 \pm 0.4$ & $36.1 \pm 0.4$ \\
\hline
\end{tabular}

aEach value is the average of three experiments; ${ }^{\mathrm{b}}$ This data refers to the paper (Yao et al., 2006).

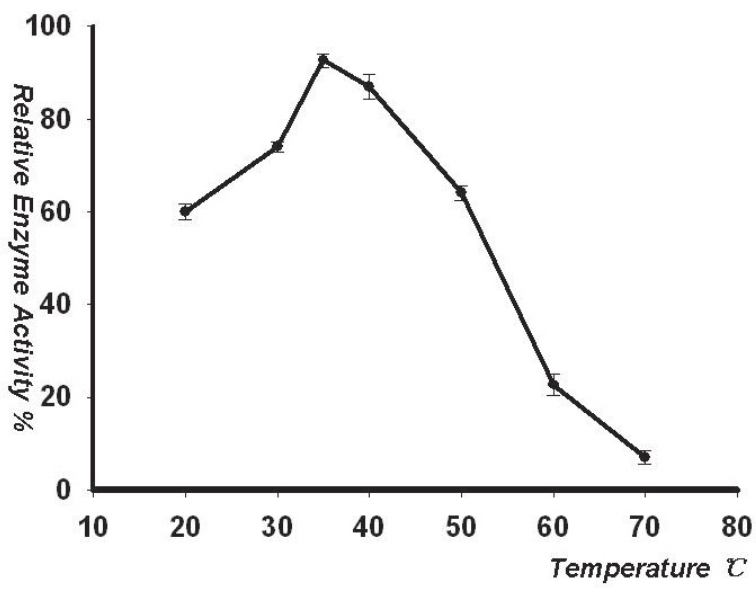

Figure 5. Effect of temperature on hydrolytic activity of the recombinant lipase

Temperature stability of the recombinant lipase was tested by pre-incubating the enzyme at different temperatures $(20,30,35$, $40,50,60$ and $70^{\circ} \mathrm{C}$ ) for $30 \mathrm{~min}$, the residual activity was then measured as described in Material and Method. Non-incubated sample was used a reference to calculate the residual activity. Data are given as means \pm S.E., $n=3$.

\section{Hydrolysis and trans-esterification by the lipase}

A spectrophotometric assay of enzyme activity and specificity toward different $p$-nitrophenyl esters by purified SR1 lipase was performed. The result showed that SR1 hydrolytic abilities were different for different acylchain length of $p$ NPS. When the acyl chain length of the $p$-nitrophenyl esters increased from $C_{10}$ to $C_{18}$, the activity decreased accordingly. The hydrolytic activity of SR1 was $556.30 \pm 2.8 \mathrm{U} / \mu \mathrm{g}$ when $p \mathrm{NPC} 10$ was used as the substrate, and $25.99 \pm 1.1 \mathrm{U} / \mu \mathrm{g}$ for $p \mathrm{NPC} 18$ substrate (Table 1). Compared with control CALB and SSDB1, hydrolytic activity of SR1 was about 28 and 40 times higher, respectively, when $p$ NPC10 was used as the substrate. This result suggested that the crude recombinant lipase has potential application in digestion of lipids especially medium-chain length fatty acids.

\section{$\mathrm{pH}$ and thermo-stability of SR1 lipase}

The thermo-stability was investigated by pre-incubating the purified enzyme in the same buffer as described in materials and methods for $30 \mathrm{~min}$ at $20^{\circ} \mathrm{C}$ to $70^{\circ} \mathrm{C}$ and then the mixture was used to determine its hydrolytic activity. The purified recombinant lipase activity was the highest at $35^{\circ} \mathrm{C}-40^{\circ} \mathrm{C}$ (Fig. 5). As shown in Fig. 5, the residual lipase activity still amounted to $97 \%$ and $95 \%$ of the control after treatment at $20^{\circ} \mathrm{C}$ and $30^{\circ} \mathrm{C}$ for $30 \mathrm{~min}$, respectively. Figure 5 also reveals that the enzyme was inactivated rapidly at temperatures higher than $50^{\circ} \mathrm{C}$ and was inactivated totally at $70^{\circ} \mathrm{C}$ within $30 \mathrm{~min}$.

The purified recombinant lipase activity was measured at various pHs in buffers with the same ionic concentrations. $\mathrm{pH}$ stability was tested by a 2 -h pre-incubation of the crude recombinant lipase in appropriate buffers that had the same ionic concentrations at different $\mathrm{pH}$ values ranging from 4.0 to 10.0 at $37^{\circ} \mathrm{C}$. Our results (Fig. 6) show that the maximum activity was observed at $\mathrm{pH}$ 7.5-8.0. The lipase activity decreased immediately when the $\mathrm{pH}$ 


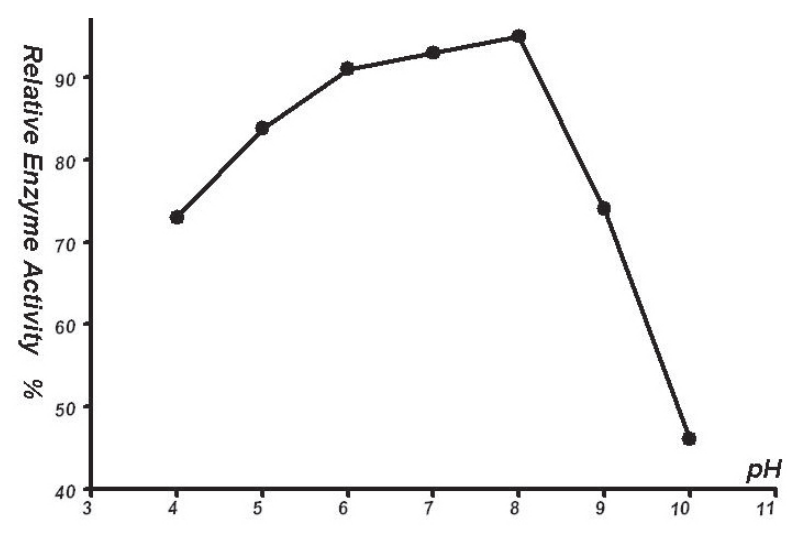

Figure 6. Effect of $\mathrm{pH}$ on hydrolytic activity of the recombinant lipase

$\mathrm{pH}$ Stability was tested by a 2 -h pre-incubation of the recombinant enzyme in appropriate buffers that had the same ionic concentrations at different $\mathrm{pH}$ values ranging from 4.0 to 10.0 at $37^{\circ} \mathrm{C}$. The remaining activity was measured immediately after this treatment with the standard method as mentioned in the text. Values are given as mean \pm S.E., $n=3$.

exceed 8.0, while the enzyme was stable in the $\mathrm{pH}$ range from 5.5 to 8.0 .

\section{DISCUSSION}

The aim of this study was to clone a lipase with a high hydrolytic activity to degrade oil in the contaminated niche. Since in oil-contaminated soil a large microbial diversity is encountered, it is difficult to obtain pure microorganism cultures (Jiang et al., 2006). Meta-genomic library was then constructed and used to screen lipase gene (Helen et al., 2005). The SR1 gene encoding an extracellular lipase cloned from the meta-genomic library was composed of $2133 \mathrm{bp}$ in length while the ORF was consisted of $1845 \mathrm{bp}$. The predicted protein consisted of 615 amino acids with a calculated molecular mass of $65 \mathrm{kDa}$. Nucleotide sequence alignment showed that SR1 gene has 98\% identity with S. marcescens lipase except ten single-nucleotide substitutions. The amino- acid sequence was also found to be more closely related to S. marcescens than to the lipases from other bacteria like Pseudomonas (not shown). In this study, we also cloned five lipase genes from Pseudomonas (four lipase genes) and Serratia, besides the SR1 gene. These genes are the same as those lipase genes described earlier (Gao et al., 1998; Jinwal et al., 2003; Gangwar et al., 2009).

Sequence analysis showed that the SR1 protein lacks an N-signal peptides and has ABC-type secretion system. Compared with other reported lipases from Serratia, the SR1 protein has similar structure including glycine-rich motif and aspartate boxes which contributed to its extracellular secretion. Sequence analysis also showed that there is only one cysteine in the SR1 protein, which is similar to other reported lipase (Li et al., 1995). Cysteines are often involved in the formation of disulfide bonds in proteins, and proteins without cysteines are generally more flexible because of the lack of disulfide bonds $(\mathrm{Li}$ et al., 1995). This characteristic is important for protein secretion of extracellular bacterial proteins, which also readily allow the conformational change that accompanies interfacial activation.

The SR1 protein had a high hydrolytic activity without a trans-esterfication activity. SR1 had not significant chain-specificity, although it had a preference for short chain substrates (C10-C12 fatty acid oil). It also displayed high hydrolytic activity for long-chain fatty acids. Compared with our previously reported SLLipA from Serratia liquefaciens S33 DB-1, the SR1 protein had a much higher hydrolytic activity for fatty acids of different length acyl. This characteristic may result from continued selection pressure, favoring mutations enhancing the catalytic efficiency of the lipase enzyme in the contaminated environment. Optimal $\mathrm{pH}$ and temperature of the recombinant SR1 lipase was $5-8^{\circ} \mathrm{C}$ and $35-40^{\circ} \mathrm{C}$, respectively. This may be due to the environment from which the gene was isolated. In the Southern China like Shanghai the soil is acidic and temperature is always high $\left(20^{\circ} \mathrm{C}-40^{\circ} \mathrm{C}\right)$. Several lipases which have been isolated from soil recently favor acidic or alkaline environment, depending on the soil $\mathrm{pH}$. Their catalytic characteristics may accelerate the process of fatty acid degradation in the soil (Lee et al., 2004; Ni et al., 2007).

In conclusion, we reported a lipase gene encoding an enzyme with non-specific hydrolysis activity. It could be applied as lipase biosensor for digestion of lipids in the food and medicine, and for oil-contamination treatment. The biological source of the SR1 gene will be determined in a future study.

\section{Acknowledgements}

This research was financially supported by Chinese National "863" High-Tech Program, No. 2007AA05Z452.

\section{REFERENCES}

Akatsuka H, Kawai E, Omori K, Komatsubara S, Shibatani T, Tosa T (1994) The lipA gene of Serratia marcescens which encodes an extracellular lipase having no N-terminal signal peptide. J Bacteriol 176: 1949-1956.

Anderson EM, Larsson KM, Kirk O (1998) One biocatalyst-many applications: the use of Candida antarctica B-lipase in organic synthesis. Biocatal Biotransform 16: 181-204.

Cervantes-González E, Rojas-Avelizapa NG, Cruz-Camarillo R, GarcíaMena J, Rojas-Avelizapa LI (2004) Oil-removal enhancement in media with keratinous or chitinous wastes by hydrocarbon-degrading bacteria isolated from oil-polluted soils. Environ Technol 29: 171-182.

Choo DW, Kurihara T, Suzuki T, Soda K, Esaki NA (1998) Coldadapted lipase of an alaskan psychrotroph Pseudomonas sp. strain B11-1: gene cloning and enzyme purification and characterization. Appl Environ Microbiol 64: 486-491.

Feller G, Thiry M, Arpigny JL, Gerday C (1991) Cloning and expression in Escherichia coli of three lipase-encoding genes from the psychrotrophic antarctic strain Moraxella TA144. Gene 102: 111-115.

Gabor EM, de Vries EJ, Janssen DB (2004) Construction, characterization, and use of small-insert gene banks of DNA isolated from soil and enrichment cultures for the recovery of novel amidases. Environ Microbiol 6: 948-958.

Ghanem A, Aboul-Enein HY (2004) Lipase-mediated chiral resolution of racemates in organic solvents. Tetrahedron: Asymmetry 15: 33313351.

Ghanem EH, Al-Sayeed HA, Saleh KM (2000) An alkalophilic thermostable lipase produced by new isolate of Bacillus alcalophilus. World J Microbiol Biotech 16: 459-464.

Gilbert EJ, Drozd JW, Jones CW (1991) Physiological regulation and optimization of lipase activity in Pseudomonas aeruginosa EF2. J Gen Microbiol 137: 2215-2221.

Gupta R, Gupta N, Rathi P (2004) Bacterial lipases: an overview of production, purification and biochemical properties. Appl Microbiol Biotech 64: 763-781.

Hasan F, Shah AA, Hameed A (2006) Industrial applications of microbial lipases. Ensyme Microbial Technol 39: 235-251.

Helen LS, Streit WR (2005) Metagenomics: Advances in ecology and biotechnology. FEMS Microbiol Lett 247: 105-111.

Henne A, Schmitz RA, BÖmeke M, Gottschalk G, Daniel R (2000) Screening of environmental DNA libraries for the presence of genes conferring lipolytic activity on Escherichia coli. Appl Environ Microbiol 66: 3113-3116.

Jaeger KE, Reetz MT (1998) Microbial lipases form versatile tools for biotechnology. Trends Biotech 16: 396-403. 
Jaeger KE, Dijkstra BW, Reetz MT (1999) Bacterial biocatalysts: molecular biology, three-dimensional structure and biotechnological applications of lipases. Annu Rev Microbiol 53: 315-351.

Jaeger KE, Ransac S, Dijkstra BW, Colson C, van Heuvel M, Misset O (1994) Bacterial lipases. FEMS Microbiol Rev 15: 29-63.

Jiang ZB, Wang HP, Ma YS, Wei DZ (2006) Characterization of two novel lipase genes isolated directly from environmental sample. Appl Microbiol Biotechnol 70: 327-332.

Kim JT, Kang SG, Woo JH, Lee JH, Jeong BC, Kim SJ (2007) Screening and its potential application of lipolytic activity from a marine environment: characterization of a novel esterase from Yarrowia lipolytica CL180. Appl Microbiol Biotechnol 74: 820-828.

Lee MH, Lee CH, Oh TW, Song JK, Yoon JH (2006) Isolation and characterization of a novel lipase from a metagenomic library of tidal flat sediments: evidence for a new family of bacterial lipases. Appl Microbil Biotechnol 72: 7406-7409.

Lee SW, Won K, Lim HK, Kim JC, Choi GJ, Cho KY (2004) Screening for novel lipolytic enzymes from uncultured soil microorganisms. Appl Microbiol Biotechnol 65: 720-726.

Lee SY, Rhee JS (1994) Hydrolysis of triglyceride by the whole cell of Pseudomonas putida 3SK in two-phase batch and continuous reactor systems. Biotechnol Bioengin 44: 437-443.

Li XY, Tetling SS, Winkler UK, Jaeger KE, Benedik MJ (1995) Gene cloning, sequence analysis, purification, and secretion by Escherichia coli of an extracellular lipase from Serratia marcescens. Appl Environ Microbiol 61: 2674-2680.

Liu ZQ, Li XY, Chi ZM, Wang L, Li J, Wang XH (2008) Cloning, characterization and expression of the extracellular lipase gene from Aureobasidium pullulans HN2-3 isolated from sea saltern. Antonie van Leeuwenhoek. 94: 245-255.

Luisa-Rúa M, Schmidt-Dannert C, Wahl S, Sprauer A, Schmid RD (1997) Thermo-alkalophilic lipase of Bacillus thermocatenulatus: Largescale production, purification and properties: aggregation behaviour and its effect on activity. J Biotechnol 56: 89-102.

Mazzafera E, Olsson IO, Sandberg G (1996) Degradation of caffeine and related methylxanthines by Serratia marcescens isolated from soil under coffee cultivation. Microbiol Ecol 31: 199-207.

Neumann G, Kabelitz N, Zehnsdorf A, Miltner A, Lippold H, Meyer D, Schmid A, Heipieper HJ (2005) Prediction of the adaptability of Pseudomonas putida DOT-T1E to a second phase of a solvent for economically sound two-phase bio-transformations. Appl Environ Microbiol 71: 6606-6612.

Ni X, Chi Z, Ma C, Madzak C (2007) Cloning, characterization and expression of the gene encoding alkaline protease in the marine yeast
Aureobasidium pullulans 10. Marine Biotechnol doi:10.1007/s10126-0079067-4.

Ogino H, Hiroshima S, Hirose S, Yasuda M, Ishimi K, Ishikawa H (2004) Cloning, expression and characterization of a lipase gene (lip3) from Pseudomonas aeruginosa LST-03. Mol Gen Genomics 271: 189-196.

Schmidt-Dannert C, Sztajer H, Stocklein W, Menge U, Schmid RD (1994) Screening, purification and properties of a thermophilic lipase from Bacillus thermocatenulatus. Biochim Biophys Acta 1214: 43-53.

Kiran GS, Shanmughapriya S, Jayalakshmi J, Selvin J, Gandhimathi R, Sivaramakrishnan S, Arunkumar M, Thangavelu T, Natarajaseenivasan K (2008) Optimization of extracellular psychrophilic alkaline lipase produced by marine Pseudomonas sp. (MSI057). Bioprocess Biosyst Eng 31: 483-492.

Shu ZY, Yan YJ, Yang JK, Xu L (2007) Aspergillus niger lipase: gene cloning, over-expression in Escherichia coli and in vitro refolding. Biotechnol Lett 29: 1875-1879.

Thompson JD, Higgins DG, Gibson TJ (1994) CLUSTAL W: improving the sensitivity of progressive multiple sequence alignment through sequence weighting, position-specific gap penalties and weight matrix choice. Nucleic Acids Res 22: 4673-4680.

Turner NJ (2004) Enzyme catalysed deracemisation and dynamic kinetic resolution reactions. Curr Opin Chem Biol 8: 114-119.

Winkler UK, Stuckmann M (1979) Glycogen, hyaluronate, and some other polysaccharides greatly enhance the formation of exolipase by Serratia marcescens. J Bacteriol 138: 663-670.

Yan JY, Yang JK, Xu L, Yan YJ (2007) Gene cloning, overexpression and characterization of a novel organic solvent tolerant and thermostable lipase from Galactomyces geotrichum Y05. J Mol Catalysis B: Ensymatic 49: 28-35.

Yao HY, Yu SW, Zhang LD, Zuo KJ, Ling H, Zhang F, Tang KX (2008) Isolation of a novel lipase gene from Serratia liquefaciens s33 DB-1, functional expression in Pichia pastoris and its properties. Mol Biotechnol 38: 99-107.

Gangwar P, Alam SI, Bansod S, Singh L (2009) Bacterial diversity of soil samples from the Western Himalayas, India. Can J Microbiol 55: 564-577.

Jinwal UK, Roy U, Chowdhury AR, Bhaduri AP, Roy PK (2003) Purification and characterization of an alkaline lipase from a newly isolated Pseudomonas mendocina PK-12CS and chemoselective hydrolysis of fatty acid ester. Bioorg Med Chem 211: 1041-1046.

Gao X, Zhang K, Cao S (1998) Isolation of a lipase-producing Pseudomonas strain and optimization of its fermentation conditions. Wei Sheng Wu Xue Bao. 38: 313-317 (in Chinese). 